\title{
A Baidu Map Based Group Navigation System
}

\author{
Lan $\mathrm{Yao}^{1, \mathrm{a}}$, Songyang $\mathrm{Jia}^{1, \mathrm{~b}}$ and Jin $\mathrm{Xu}^{1, \mathrm{c}}$ \\ ${ }^{1}$ College of Information Science and Engineering, Northeastern University, Shenyang, China

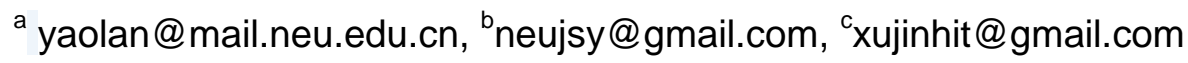

Keywords: BaiduMap API, Friends' location, group Navigation, Andriod

\begin{abstract}
In order to know friends' location and make communication more convenient especially on a trip, we develop FriendLOC system, which is an Andriod based Application for a smart phone. Baidu map is applied as a component for this development. With this system, friends' location will be shown on the map and group chat is available. In this paper, we introduce the system's developing tools, analysis the system's design process and present several achieved renderings.
\end{abstract}

\section{Introduction}

When friends drive several cars to travel, the general solution to avoid stray is to choose one car as a guide. If the guide car drives too fast to be kept up with by following cars, the problem keeps exiting. Additionally, friends intend to know each other's location during the travelling. The FriendLOC system consists of two parts: client end in cell phone and the server. With this client application we could join friends into a group and the friends' locations will be shown on the client application's map so that each car in the group knows the other cars' locations dynamically.

The rest of the paper is organized as follows. Related work is discussed in next section. Section 3 explains the whole system's architecture and functions. Section 4 presents the whole system's design and several achieved renderings. Conclusion and future work are finally given in Section 5.

\section{Related Works}

A rapidly increasing number of mobile phone users has motivated researchers from various fields to study its social and economic impact [1]. All kinds of smart mobile phone software release in endlessly and mobile map service is one of them. In recent years, due to the practicality of LBS (i.e. Location Based Service), people have paid much attention to it. It could require position information, such as get latitude and longitude. [2] develops a miniature wireless remote caller carry-on and corresponding system. When the caller pressed the emergency button, the signals immediately are sent to the campus security emergency center, at the same time, in the BaiduMap interface the caller's location is visually displayed. [3] studies the application and implementation of virtual digital campus system based on BaiduMap API. It provides detailed techniques for creating virtual campus by BaiduMap API and provides users with the synchronous position, spatial query, and navigation of the campus. [4] uses Android smart-phone platform and Baidu positioning SDK properties, with BaiduMap, designs and implements a personal positioning and route recording maps of terminal software systems based on Android smart phone platform, which is called Mobile Grid Marketing Management System.

All of these works above apply BaiduMap API to accomplish their functions. In our system, we also use BaiduMap API and our work is focusing on locating people's position and chat with others through cellular data network. 


\section{Preliminary Design}

Initialization. The whole system includes client application, server system and wireless socket communication. The client application should be installed in smart phones (we denote them as A, B and $C$ in this paper) respectively. We also apply a server with server system to communicate with client ends through wireless transmission. The architecture is shown in Fig. 1.

Socket API is applied as communication method between clients and server through cellular data network. It will open the session after a client sends connection request and closes it after the session is completed. The client uses the socket to send a connection request to the server on the network specified port. Once the connection is established successfully, it is claimed by the opened session and released when the session is completed.

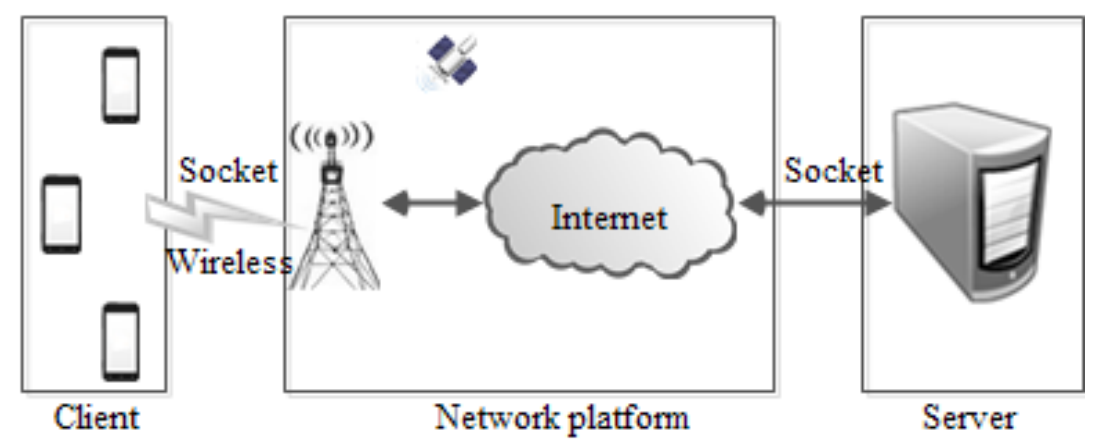

Fig. 1: Communication between clients and server

Function design. The main functions of the system is shown in Fig. 2.

\begin{tabular}{|c|c|c|c|}
\hline $\begin{array}{c}\text { join/quit } \\
\text { a } \\
\text { group }\end{array}$ & $\begin{array}{c}\text { send/receive } \\
\text { chat } \\
\text { message }\end{array}$ & $\begin{array}{c}\text { send/receive } \\
\text { location } \\
\text { information }\end{array}$ & $\begin{array}{c}\text { plot } \\
\text { location } \\
\text { on map }\end{array}$ \\
\hline
\end{tabular}

Client

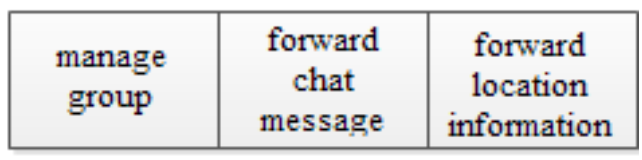

Server

Fig. 2: Function design

On a client, the following functions are designed:

i) Register. The user registers with phone number as a unique identity.

ii) Join or quit a group. The user could create a group, invite others to join the group and dissolve the group. Users also could join in or quit the others' group.

iii) Send or receive chat massages.

iv) Send or receive location information.

v) Plot location on map dynamically.

On the server, the following functions are designed:

i) Manage users.

ii) Manage groups.

iii) Forward chat messages.

iv) Forward location information.

The developing tools are shown in Fig. 3. We develop the client part based on Android operating system with Java. We use Eclipse integrated with Android SDK (i.e. Software Development Kit) and ADT (i.e. Android Development Kit). We call BaiduMap Application Programming Interface (API) directly as the localization tool. 


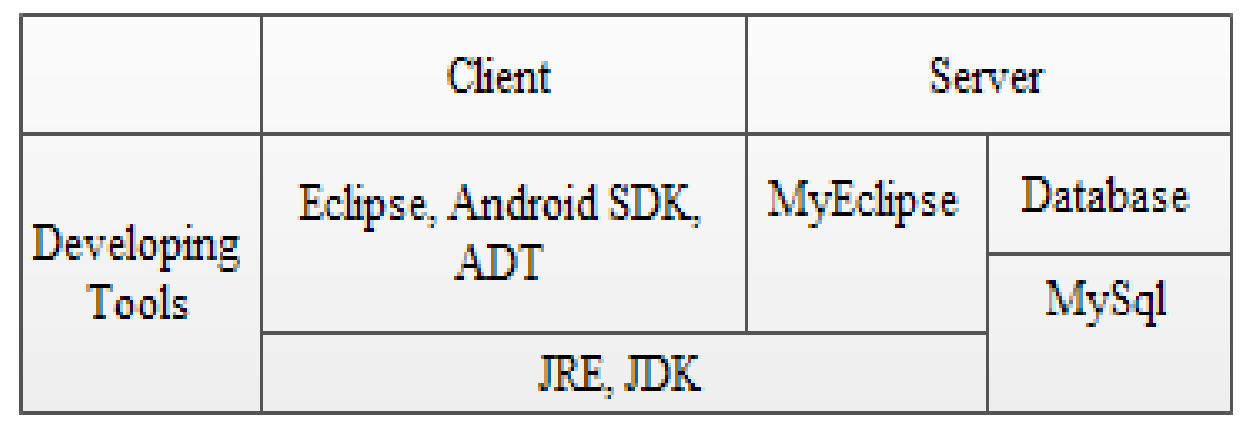

Fig. 3: Developing tools

\section{FriendLOC Design and Implementation}

System Architectural Design. The system architectural is shown in Fig. 4. The communication between clients need be conducted through the server. The map needs to be displayed on the client's main interface, through cellular data network to load the map and the acquiring of the client's position is also through cellular data network or through GPS. Once the location information is sent to the other group members by the server, it will be plotted on the maps of members' cell phones.

\begin{tabular}{|c|c|c|c|c|c|c|}
\hline \begin{tabular}{|r|} 
Map \\
Baic
\end{tabular} & $\begin{array}{c}\text { Send/Receive } \\
\text { Map Information } \\
\text { duMap Android } \\
\text { SDK }\end{array}$ & $\begin{array}{l}\text { Send/Receive } \\
\text { Others Data }\end{array}$ & \multirow{2}{*}{$\begin{array}{c}\text { Cellular Data } \\
\text { Network }\end{array}$} & $\begin{array}{l}\text { Send/Receive } \\
\text { Map } \\
\text { Information } \\
\end{array}$ & $\begin{array}{l}\text { Send/Receive } \\
\text { Others Data }\end{array}$ & $\begin{array}{l}\text { Manage } \\
\text { Group }\end{array}$ \\
\hline \multicolumn{3}{|c|}{ Socket API } & & \multicolumn{3}{|c|}{ Socket API } \\
\hline \multicolumn{3}{|c|}{ Android } & & & & \\
\hline
\end{tabular}

Client

Server

Fig. 4: System architecture

Server Design. The design pattern of server adopts layered architecture. The server architecture is divided into four layer as shown in Fig. 5. Each layer are described below.

i) Network Interface Layer. Network interface layer provides business service for the interaction between the server and the client. The communication between the server and client is based on Socket API and adopts TCP protocol to transfer messages.

After the server network interface layer receives the data from a client, it parses the data's type and calls the business treatment layer corresponding processing functions according to the data type. The network interface layer encapsulates the data to Socket data stream according to the custom data structure type, then send the Socket data stream to the client.

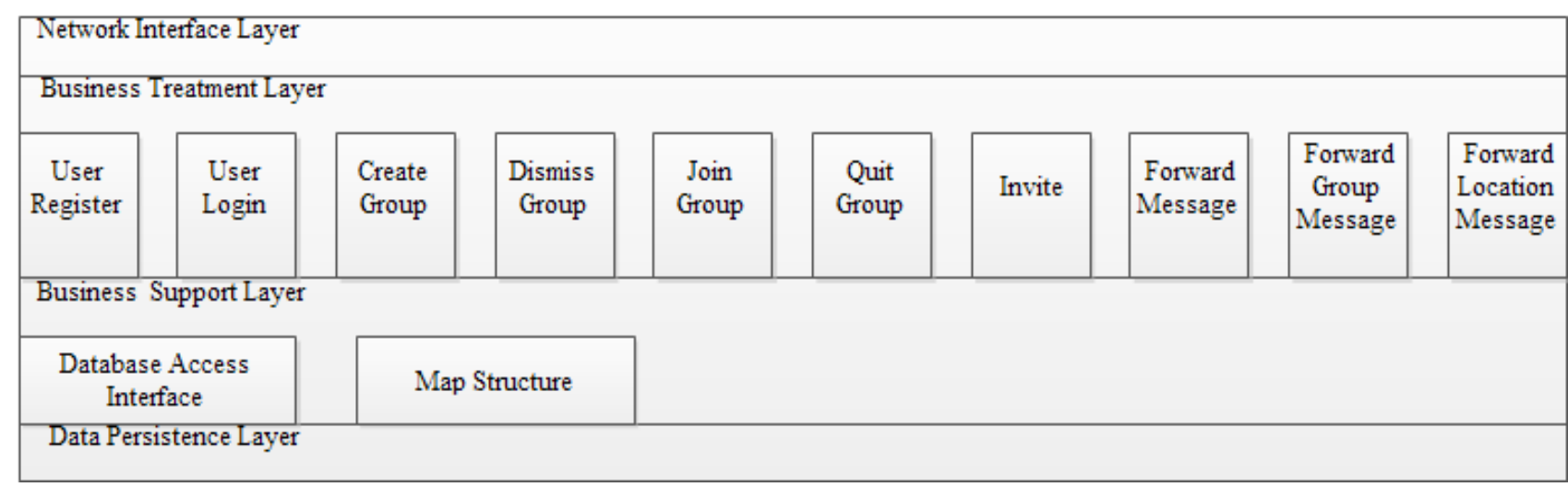

Fig. 5: Server layered architecture 
ii) Business Treatment layer. The business treatment layer processes specific business from client. The processing functions in business treatment layer are called by network interface layer. The processing results are returned to network interface layer.

The functions that need to be processed by business treatment layer include: Register, Login, Create a group, Dismiss a group, Join a group, Quit a group, Invite someone join a group, Forward messages, Forward group messages, Forward location messages, etc.

iii) Business Support Layer. Business support layer provides the tools of business treatment for business treatment layer.

Forwarding location message request needs to record all the online users' current positions, a group ID and iterate over to generate a data structure that is from one-to-one correspondence structure of "User ID : location information" pairs for all the online users. For forwarding location request is for all the online users, these lists need to store in the database.

iv) Data Persistence Layer. At the data persistence layer, data is stored in the hardware to keep it persistent. In the server, data persistence layer refers to the database.

Client Design. The client architecture is divided into four layers, which are presentation layer, map manipulating layer, business request layer and network interface layer, as shown in Fig. 6. The conception and functions of each layer are described in the following sections. Each layer are described below.

\begin{tabular}{|c|c|c|c|c|c|c|c|c|c|}
\hline \multicolumn{10}{|c|}{ Presentation Layer } \\
\hline \multicolumn{2}{|c|}{ Map } & \multicolumn{2}{|c|}{ Login } & \multicolumn{2}{|c|}{ Register } & \multicolumn{2}{|c|}{ Chat } & \multicolumn{2}{|c|}{ Group Manager } \\
\hline \multicolumn{10}{|c|}{ Map Manipulate Layer } \\
\hline \multicolumn{2}{|c|}{ Real Time Locate } & \multicolumn{2}{|c|}{ Browse Maps } & & & & & & \\
\hline \multicolumn{10}{|c|}{ Business Request Layer } \\
\hline $\begin{array}{c}\text { User } \\
\text { Register }\end{array}$ & $\begin{array}{l}\text { User } \\
\text { Login }\end{array}$ & $\begin{array}{l}\text { Create } \\
\text { Group }\end{array}$ & $\begin{array}{c}\text { Dismiss } \\
\text { Group }\end{array}$ & $\begin{array}{l}\text { Join } \\
\text { Group }\end{array}$ & $\begin{array}{l}\text { Quit } \\
\text { Group }\end{array}$ & Invite & $\begin{array}{l}\text { Send or } \\
\text { Receive } \\
\text { Message }\end{array}$ & \begin{tabular}{|c|} 
Send or \\
Receive \\
Group \\
Message
\end{tabular} & \begin{tabular}{|l} 
Send or \\
Receive \\
Location \\
Message
\end{tabular} \\
\hline \multicolumn{10}{|l|}{ Network I } \\
\hline
\end{tabular}

Fig. 6: Client layered architecture

i) Presentation Layer. Presentation layer is the input and output interface of the client, ie. the interaction interface between the client and users.

In the client, the presentation layer includes the following interfaces: map, login, register, chat, group manager, etc.

The map interface includes map layer, location layer, friends' position layer. Map layer displays the planar map information, location layer displays the client's real time position information and friends' position layer displays the current online group members' real time position information. These layers can be superimposed: the upper layer data will cover the lower layer data.

The other interface needs user to type in corresponding data, then the client will jump to corresponding interface or display the corresponding content on the client.

ii) Map Manipulate Layer. The manipulation of the client map manipulating layer includes map browse and real time localization.

The manipulating object of map browse is the map layer in presentation layer's map interface It allows you to zoom in and out. The localization requires the client's current longitude and latitude, generates location layer and overlaps the map layer. 


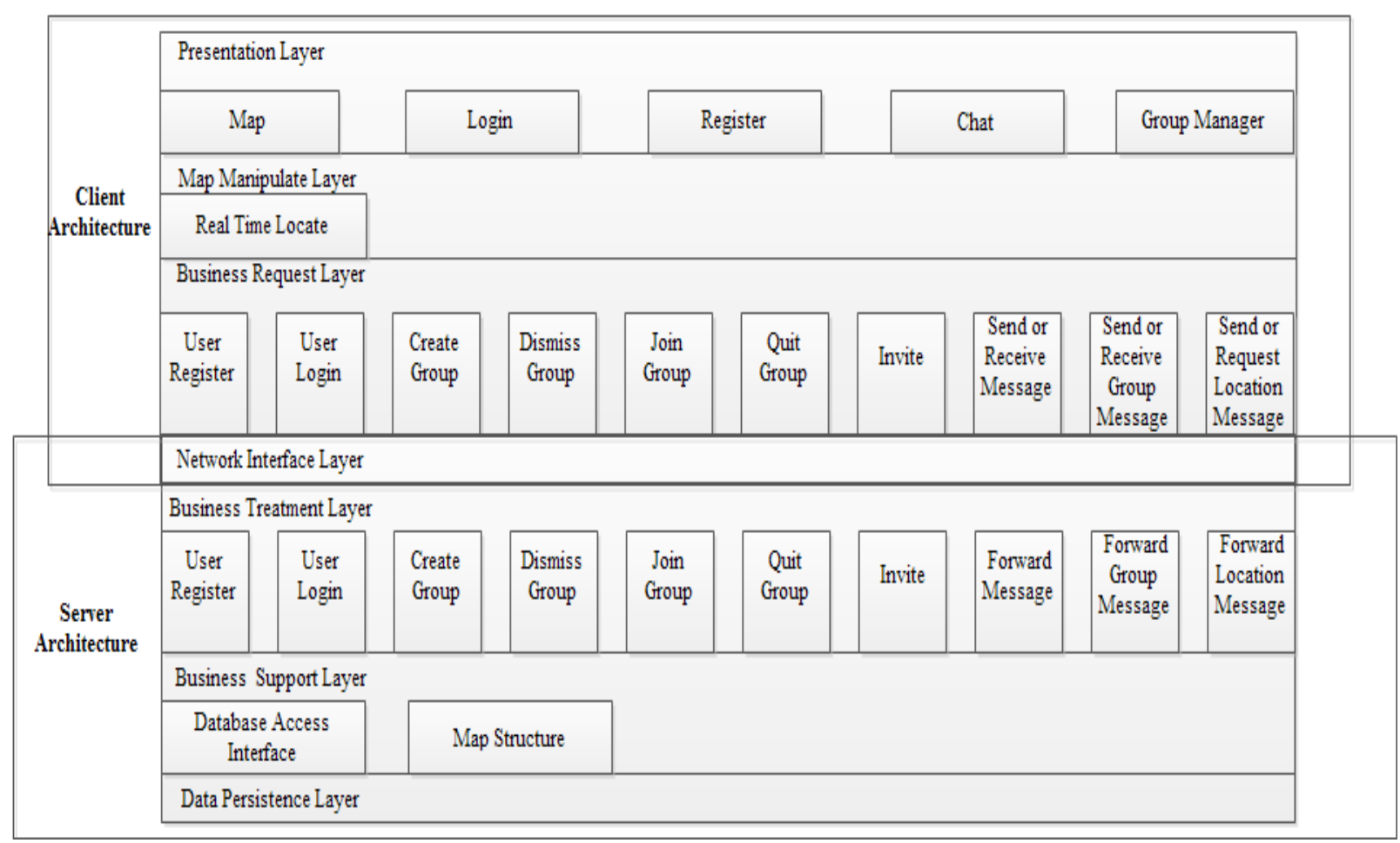

Fig. 7: The sketch of interacting layer between the client and the server

iii) Business Request Layer. The server is the core of processing and realization. The client requests the server to provide business service. It need to interact data with the server. In the client architecture, business request layer takes charge of request business service to the server and process the response result that returns from the server.

The business request in client business request layer includes Register, Login, Create a group, Dismiss a group, Join in a group, Quit a group, Invite someone to join a group, Send or Receive messages, Send or Receive group Messages, Send or Receive Location Messages.

iv) Network Interface Layer. Network interface layer is the interface of requiring business service and communication between the client and server. The network interface layer is the interacting layer between the client and the server. The Socket request message that sent by network interface layer in client is received and decapsulated by the network interface layer in server. The Socket response message that returns from the network interface layer is received and decapsulated by the network interface layer in client. The sketch of interacting layer between the client and the server is shown in Fig. 7.

The network interface layer in client encapsulates the request messages from business request layer to custom message and sends them to the server. Then it receives the response from the server. After the network interface layer in the client receives the Socket response message from the server, it decapsulates the response message and passes it to business request layer. Then the business request layer processes the response message.

Implementation. We emphasize the accomplishment of two main functions that are group chat and friends' position display. We use three Android phones (A, B, and C) as users to accomplish this system.

A creates a group named Lets Travel and invites B and C to join the group. The UI of group chat among $\mathrm{A}, \mathrm{B}$, and $\mathrm{C}$ is shown in Fig.8. The figure is a screenshot from client $\mathrm{A}$. A sends a message in the group and $\mathrm{B}$ and $\mathrm{C}$ response a message respectively after receiving the message. 


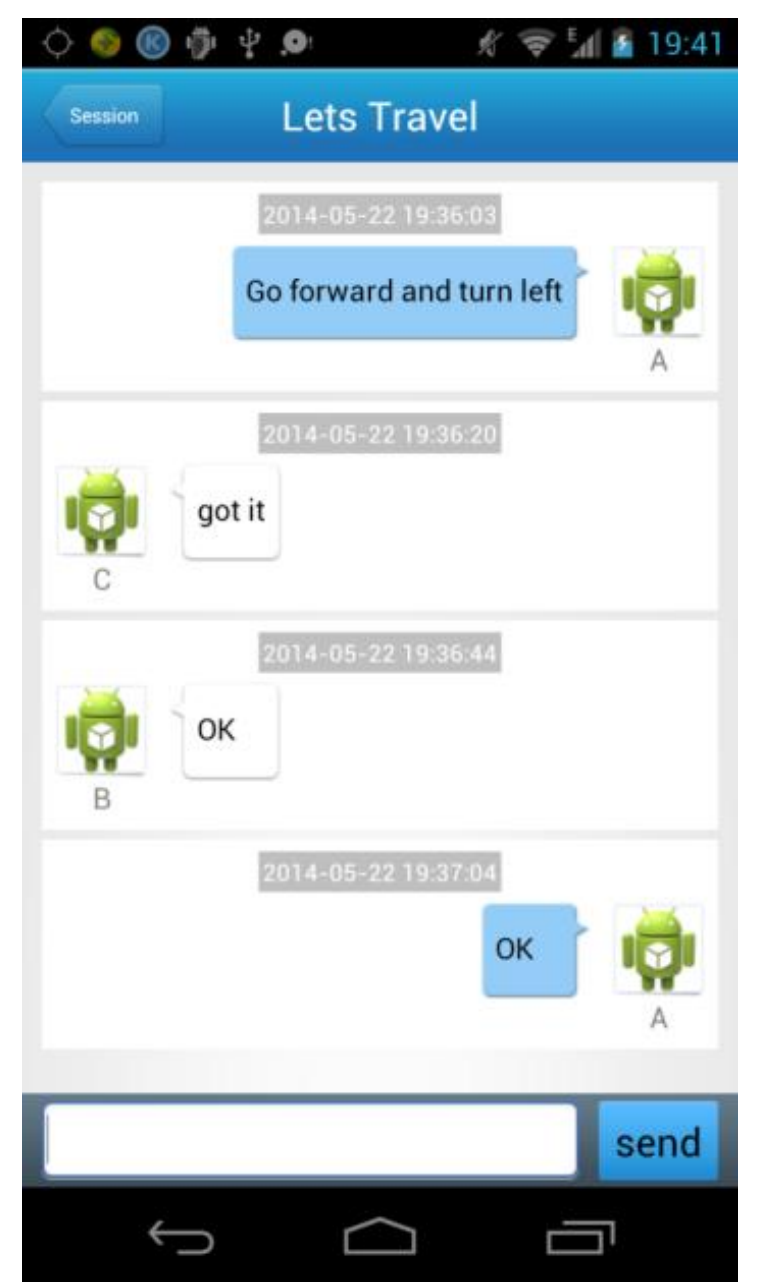

Fig. 8: Group chat

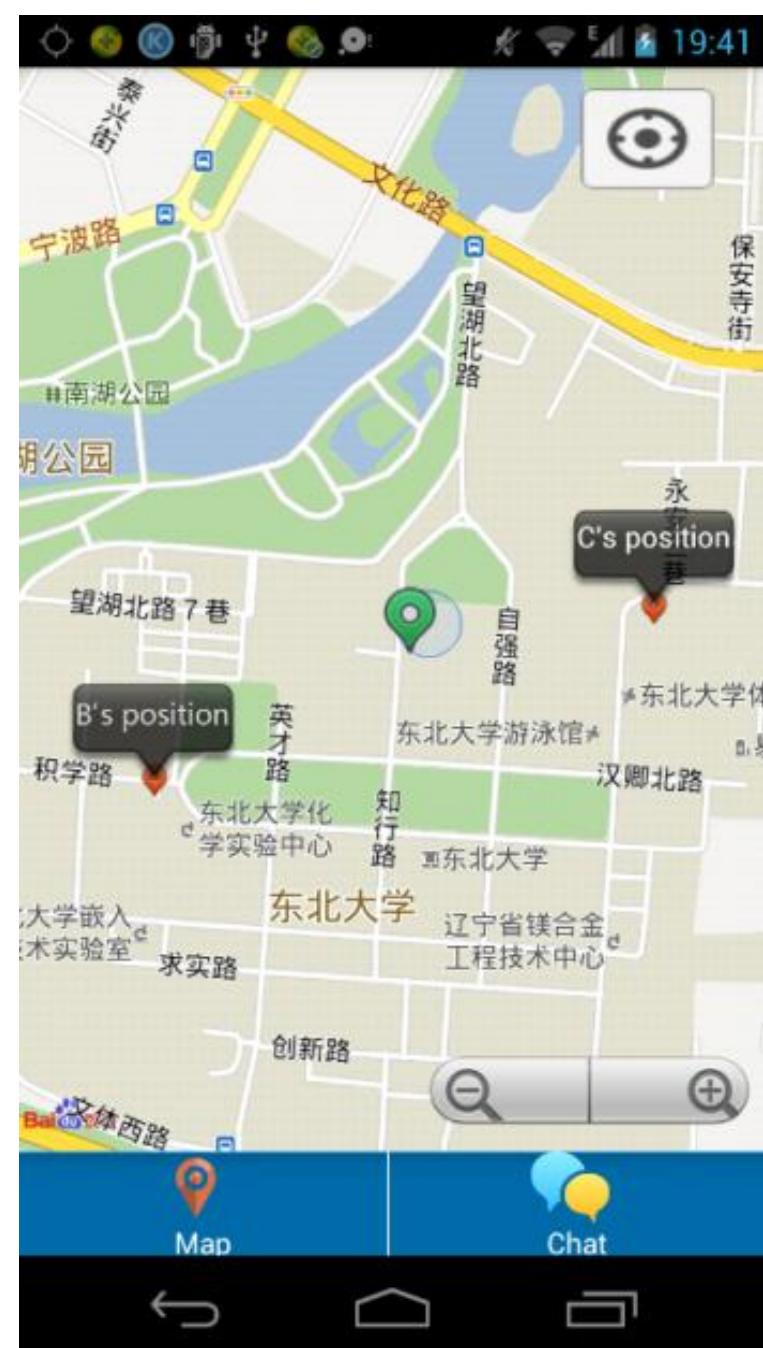

Fig. 9: Friends' location

The location of $\mathrm{A}, \mathrm{B}$, and $\mathrm{C}$ is shown in the Fig. 8. The figure is a screenshot from client $\mathrm{A}$. The green point is the host A's position, the other two red points are B and C. Click the red point, a bubble will be shown on the top of the point and tip appears on the bubble to indicate who the point is about. See Fig. 9, when click the right red point, a bubble will be shown on the point and indicates that the point is C's position.

\section{Conclusions and Future Work}

In this paper, we have developed FriendLOC system, introduced system development tools, functions, client design, server design, database's design and several achieved renderings. With this system, we could join several friends into a group when we go on a trip together. Thus we could launch a group chat or an individual chat with other group members. We also could locate every group members' position on the map while travelling. The system makes communication more convenient and localization more efficient.

As future work, we are going to enhance the system's security, such as encrypting and serve more functions of the system, such as audio chat.

\section{Acknowledgements}

This research is supported by the National Natural Science Foundation of China under Grant No. 61100182. 


\section{References}

[1] Santi Phithakkitnukoon, TeerayutHoranont, Giusy Di Lorenzo, Ryosuke Shibasaki, and Carlo Ratti: Activity-Aware Map: Identifying Human Daily Activity Pattern Using Mobile Phone Data, Lecture Notes in Computer Science Volume 6219, 2010, pp 14-25.

[2] Chen Haisheng, CaiZeming, ChenDeyi, andDengRui: Campus alarm locating system based on nRF24LE1 and BaiduMap. Microcontrollers\& Embedded Systems, 2014(2), p.73.

[3] Liang Guangrong, and Li Hong: Baidu Map API Based Design and Development of Virtual Campus. Journal of Guangdong Peizheng College, 2012(2), p.85.

[4] Li Ruixuan, Wang Shandong, Xu Zhiyuan, and Wang Lingli: Design and realization of positioning system based on Android platform. Information Technology, 2013(12), p.187. 